\author{
'Cartas científicas \\ Rev Urug Cardiol 2021; 36: e702 \\ doi: $10.29277 /$ cardio.36.3.14
}

\title{
Cuando no todo es lo que parece... Masa extracardíaca en la aurícula izquierda: reporte de un caso en Uruguay
}

\section{Resumen}

La hernia hiatal es una entidad relativamente frecuente y puede ser un hallazgo incidental en un estudio ecocardiográfico. Describimos el caso de una paciente con diagnóstico de masa en la aurícula izquierda (AI) sin clara etiología, a quien se le realiza el diagnóstico de hernia hiatal por resonancia magnética cardíaca (RMC) y luego se demuestra mediante ecocardiografía de contraste el contenido gástrico de la masa tras la ingestión de una bebida carbonatada, lo que permite de forma rápida y sencilla aclarar el diagnóstico.

Palabras clave: HERNIA HIATAL

MULTIMODALIDAD DE IMAGEN

When not everything is what it seems... Extra-cardiac mass in the left atrium: a case report in Uruguay

\section{Summary}

Hiatal hernia is a relatively common entity, and may be an incidental finding in an echocardiographic study. We describe the case of a patient with a diagnosis of a mass in the left atrium with no clear etiology, in whom the diagnosis of hiatal hernia is made by cardiac magnetic resonance imaging and then demonstrated by contrast echocardiography the gastric content of the mass after the ingestion of a carbonated drink, which allows quickly and easily to clarify the diagnosis.

Key words: $\quad$ HIATAL HERNIA

IMAGE MULTIMODALITY

\section{Quando nem tudo é o que parece... Massa extracardíaca em átrio esquerdo: relato de um caso no Uruguai}

\section{Resumen}

A hérnia de hiato é uma entidade relativamente comum, e pode ser um achado incidental em um estudo ecocardiográfico. Descrevemos o caso de um paciente com diagnóstico de massa em átrio esquerdo sem etiologia definida, em que o diagnóstico de hérnia de hiato é feito por ressonância magnética cardíaca e posteriormente demonstrado por ecocardiografia contrastada com uma bebida gaseificada, permitindo esclarecer de forma rápida e fácil o diagnóstico. Palavras chave: HÉRNIA DE HIATO MULTIMODALIDADE DE IMAGEM

Sr. Editor:

\section{Introducción}

La hernia hiatal es una entidad relativamente frecuente, caracterizada por el desplazamiento de la unión gastroesofágica, el estómago y/u otras vísceras abdominales hacia el mediastino. Aunque puede producir síntomas sugestivos de etiología cardiovascular, solo se han detectado pocos casos por su manifestación ecocardiográfica. Frente a la sospecha de una masa que comprime la AI debemos considerar la hernia hiatal como un diagnóstico válido y mediante técnicas sencillas complementarias del ecocardiograma o técnicas avanzadas, como la resonancia, se puede llegar a su diagnóstico y sus diferenciales ${ }^{(1)}$. Presentamos el caso de una paciente con diagnóstico de masa en la AI durante largo tiempo que se la envía a RMC para esclarecer su etiología, evidenciándose un gran saco herniario con desplazamiento de esta, la cual corroboramos en el ecocardiograma de forma rápida y sencilla como describiremos.

\section{Caso clínico}

Paciente de sexo femenino de 67 años, con hábito pícnico (peso $90 \mathrm{~kg}$, talla $150 \mathrm{~cm}$ ) y antecedentes de hipertensión arterial en tratamiento con losartán $50 \mathrm{mg} /$ día. Historia de meses de evolución de disnea de esfuerzo CF II sin causa clara. Historia de dispepsia y episodios de reflujo gastroesofágico. ECG en ritmo sinusal $78 \mathrm{cpm}$, QRS fino, sin alteraciones de la repolarización. Presentaba ecocardiogramas que eran similares desde hace tres años donde se informa una masa que comprime y/o 


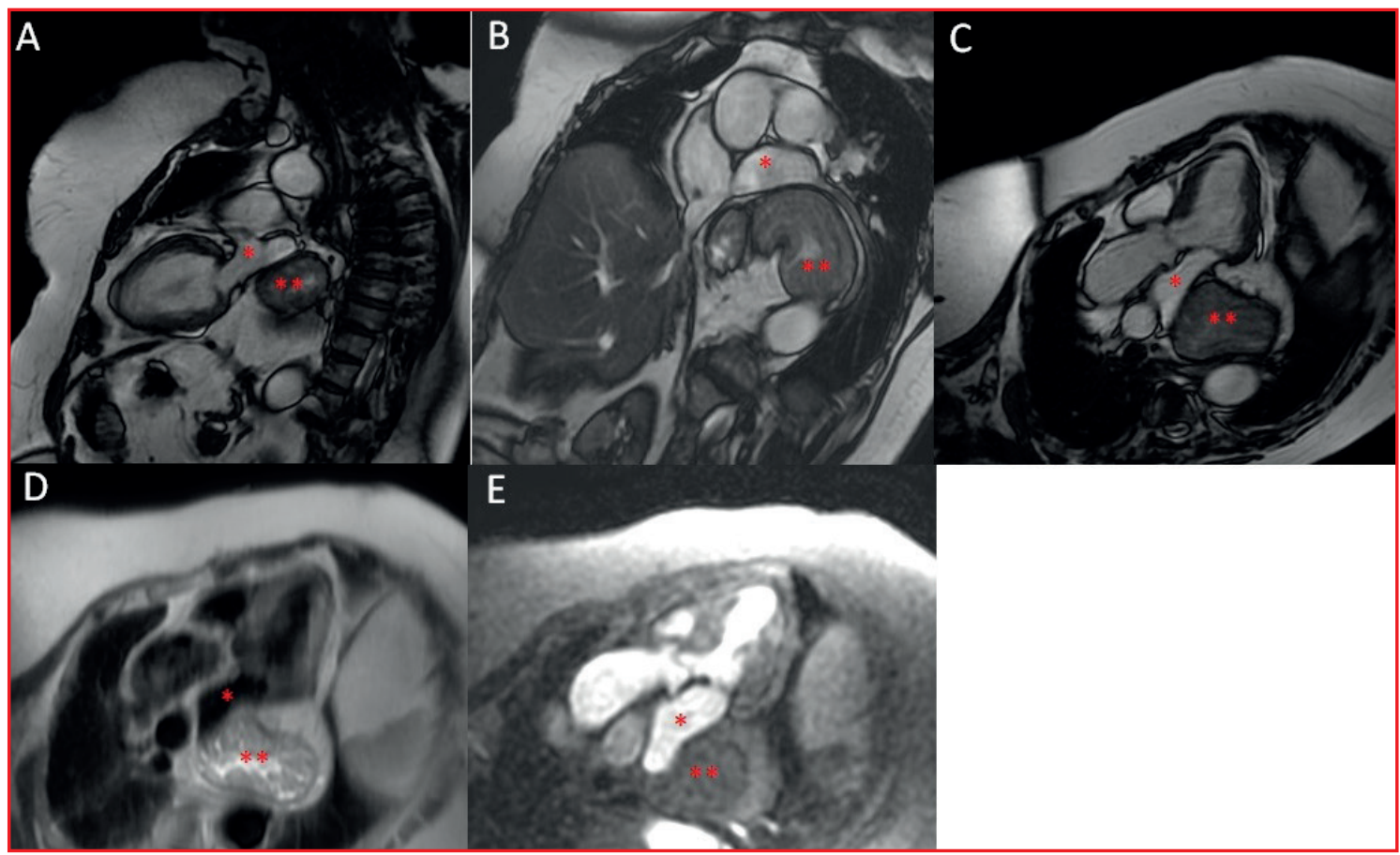

Figura 1. Resonancia magnética cardíaca. A-B-C) Secuencias de cine donde se puede observar una masa extracardíaca en el sector posterior de la aurícula izquierda $\left({ }^{*}\right)$ que corresponde a la cavidad gástrica $\left(^{* *}\right)$, compatible con hernia hiatal. D) Secuencia ponderada en T2. E) Secuencia de perfusión.

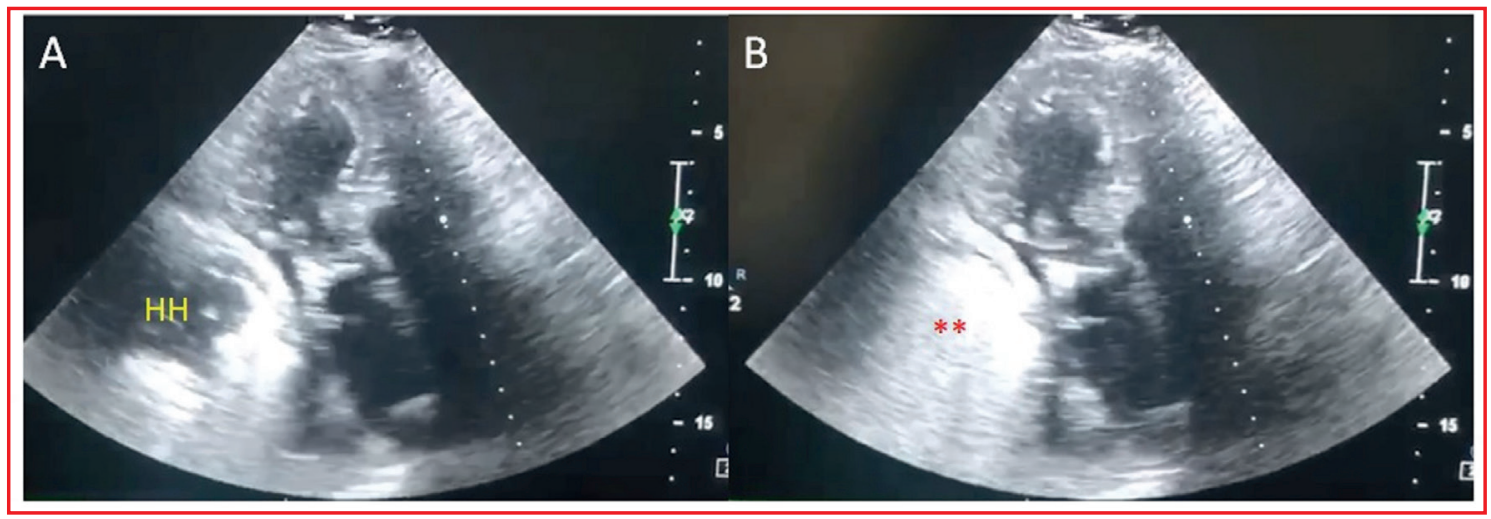

Figura 2. Ecocardiograma doppler en vista apical eje largo. A) Se observa una masa anecoica en su interior que corresponde a una hernia hiatal $(\mathrm{HH})$ en el sector posterior de la aurícula izquierda y pared inferolateral del ventrículo izquierdo. B) Luego de que la paciente ingiere una bebida carbonatada (agua con gas) se observa como las burbujas pasan al interior del estómago $(* *)$ lo que confirma la presencia de hernia hiatal mediante ecocardiografía.

desplaza la AI sin poder dilucidar con claridad su etiología, asociado a una mala ventana ultrasónica. Por esta razón es enviada por su médico tratante para realización de una RMC con el fin de esclarecer el diagnóstico. La resonancia informa ventrículo izquierdo con volúmenes de fin de diástole y fin de sístole normales, FEVI conservada y destaca la presencia de una masa extracardíaca en el sector posterior de la AI, que desplaza y comprime parcialmente a esta y la vena pulmonar inferior izquierda; por características morfológicas y caracterización tisular corresponde a estómago.
En suma, gran hernia hiatal con desplazamiento y compresión parcial de AI y vena pulmonar inferior izquierda (figura 1).

Luego, con este planteo se realiza un nuevo ecocardiograma Doppler con el fin de contar con una técnica de mayor accesibilidad para un eventual seguimiento cardiovascular. En este se constata una regular ventana ultrasónica y se evidencia una masa retroauricular en íntimo contacto con la aurícula izquierda y sobre pared inferolateral del ventrículo izquierdo de difícil caracterización (figura 2A). Contando con la información de la reso- 
nancia se completa el estudio ecocardiográfico y se le pide a la paciente que ingiera una bebida carbonatada en decúbito supino (en este caso, agua con gas, a modo de "contraste"). Se visualiza el pasaje de las microburbujas al interior de la masa (en este caso, el estómago), lo que permite confirmar el diagnóstico ecocardiográfico de hernia hiatal (figura 2B). Dados estos hallazgos en los estudios de multimodalidad de imagen cardiovascular, es enviada por su médico tratante a valoración por equipo de gastroenterología y cirugía para definir directivas del tratamiento a seguir.

\section{Discusión}

La hernia hiatal es una entidad relativamente frecuente, caracterizada por el desplazamiento de la unión gastroesofágica, el estómago y/u otras vísceras abdominales hacia el mediastino. La manifestación clínica más característica es el reflujo gastroesofágico, que se manifiesta a través de regurgitación y pirosis. Sin embargo, aunque menos frecuente, cuando es de gran tamaño puede manifestarse con síntomas de la esfera cardiovascular como disnea o dolor torácico, síncope, sobre todo posprandial y luego de una comida copiosa, así como arritmias y alteraciones electrocardiográficas secundarias a la compresión cardíaca, como se ha reportado en la literatura ${ }^{(2)}$.

El diagnóstico de hernia hiatal puede ser a veces un gran desafío debido al cambio en la anatomía de la unión gastroesofágica durante la deglución, la respiración y el movimiento. Es obligatorio realizar una historia clínica y examen físico completos, ya que pueden revelar síntomas que antes no eran evidentes. Cuando una hernia hiatal es demasiado grande, el desplazamiento del contenido abdominal en la cavidad torácica puede causar compresión de la AI, que puede ser un hallazgo incidental durante un estudio ecocardiográfico. Si no se tiene alta sospecha, su diagnóstico por ecocardiografía puede pasar desapercibido. El diagnóstico diferencial de una masa que invade la AI incluye la hernia de hiato, aneurismas de la aorta torácica descendente, carcinoma esofágico, diseminación de carcinoma broncogénico y otras masas mediastínicas posteriores. Se ha descrito que la hernia hiatal puede simular una masa intraauricular o una estructura mediastí- nica posterior en la ecocardiografía transtorácica. Nishimura y col. ${ }^{(3)}$ en 1985 fueron los primeros en informarlo en imágenes ecocardiográficas bidimensionales $^{(4)}$

La ingestión de bebidas gaseosas para el diagnóstico ecocardiográfico de hernia hiatal se ha informado ocasionalmente en la literatura ${ }^{(1)}$. Algunos autores han reportado la utilidad de cócteles basados en la combinación de una bebida carbonatada mezclada con medio de contraste ecocardiográfico lo que permite la visualización de las burbujas en la víscera abdominal y una mejor delimitación de la hernia, confirmando el diagnóstico ecocardiográfico ${ }^{(5,6)}$. En nuestra experiencia, la simple ingestión de una bebida carbonatada, como agua con gas, puede ser suficiente para dilucidar el diagnóstico ante la sospecha de hernia hiatal por ecocardiografía y técnicas de imagen más avanzadas, como la resonancia, pueden marcar el diagnóstico diferencial.

En cuanto al tratamiento de una hernia hiatal sintomática, el objetivo primario es reducir los síntomas de reflujo gastroesofágico comenzando por cambios en el estilo de vida como perder peso, elevar la cabecera de la cama, evitar acostarse inmediatamente después de una comida, eliminar alimentos desencadenantes como el alcohol, el café, el chocolate, las comidas picantes, etc. El tratamiento médico se basa, sobre todo, en inhibidores de la bomba de protones, antagonistas del receptor de histamina y antiácidos. El tratamiento quirúrgico, si bien depende del tipo de hernia, en general queda reservado para pacientes que persisten sintomáticos a pesar del tratamiento médico.

\section{Conclusiones}

La hernia hiatal se puede presentar tanto clínica como ecocardiográficamente, simulando una patología cardiovascular. Aunque el diagnóstico definitivo suele confirmarse mediante otras técnicas de imagen, existen hallazgos ecocardiográficos que orientan fuertemente al diagnóstico. Dada su facilidad técnica y disponibilidad, la ecocardiografía de contraste oral con una bebida carbonatada puede ser una herramienta de gran ayuda para aclarar el diagnóstico ante una masa en la AI de origen desconocido y sospecha de hernia hiatal. 
Pablo Fontes, ORCID 0000-0001-7896-4211

Gabriel Parma, ORCID 0000-0001-6358-3723

Natalia Lluberas, ORCID 0000-0003-1400-0784

Fabián Martínez, ORCID 0000-0002-9496-4737

Julia Aramburu, ORCID 0000-0003-1221-595X

Este artículo fue aceptado para su publicación por el Editor jefe Dr. Federico Ferrando

\section{Bibliografía}

1. Gupta M, Nanda NC, Inamdar V. Two- and three-dimensional transthoracic echocardiographic assessment of hiatal hernia. Echocardiography. 2008;25(7):790-793. doi:10.1111/j.15408175.2008.00702.x

2. Maekawa T, Suematsu M, Shimada T, Masayoshi GO, Shimada T. Unusual swallow syncope caused by huge hiatal hernia. Intern Med.
2002;41(3):199-201. doi:10.2169/internalmedicine.41.199

3. Nishimura RA, Tajik AJ, Schattenberg TT, Seward JB. Diaphragmatic hernia mimicking an atrial mass: a two-dimensional echocardiographic pitfall. J Am Coll Cardiol. 1985;5(4):992-5. doi:10.1016/S0735-1097(85)80446-0

4. Lim HS, Leong DP, Alasady M. Massive hiatus hernia mimicking a left atrial mass. Hear Lung Circ. 2013;22(10):875-876. doi:10.1016/j.hlc.2013.01.001

5. Buss S, Katus HA, Mereles D. Dynamic changing mass behind the left atrium. Heart. 2007;93(12):1583. doi:10.1136/hrt.2006.107136

6. Koskinas KC, Oikonomou K, Karapatsoudi E, Makridis P. Echocardiographic manifestation of hiatus hernia simulating a left atrial mass: Case report. Cardiovasc Ultrasound. 2008;6:46. doi:10.1186/1476-7120-6-46 